\title{
Visualised Inspection System for Monitoring Environmental Anomalies During Daily Operation and Maintenance
}

\author{
Xiang XIE \\ Institute for Manufacturing, University of Cambridge \\ Cambridge CB3 OFS, UK \\ xx809@cam.ac.uk \\ Qiuchen LU (Corresponding Author) \\ Institute for Manufacturing, University of Cambridge \\ Cambridge CB3 OFS, UK \\ Bartlett School of Construction and Project Management, University College London \\ London WCIH OQB, UK \\ qiuchen.lu@ucl.ac.uk \\ David RODENAS-HERRAIZ \\ Centre for Smart Infrastructure and Construction, University of Cambridge \\ Cambridge CB3 OFA, UK \\ dr424@cam.ac.uk \\ Ajith Kumar PARLIKAD \\ Institute for Manufacturing, University of Cambridge \\ Cambridge CB3 OFS, UK \\ aknp2@cam.ac.uk \\ Jennifer Mary SCHOOLING \\ Centre for Smart Infrastructure and Construction, University of Cambridge \\ Cambridge CB3 OFA, UK \\ jms33@cam.ac.uk
}

\begin{abstract}
Purpose:

Visual inspection and human judgement form the cornerstone of daily operations and maintenance $(\mathrm{O} \& \mathrm{M})$ services activities carried out by facility managers nowadays. Recent advances in technologies such as building information modelling (BIM), distributed sensor networks, augmented reality (AR) technologies and digital twins present an immense opportunity to radically improve the way daily O\&M is conducted. This paper describes the development of an AR-supported automated environmental anomaly detection and fault isolation method to assist facility managers in addressing problems that affect building occupants' thermal comfort.
\end{abstract}

Design/methodology/approach: 
The developed system focuses on the detection of environmental anomalies related to thermal comfort of occupants within a building. The performance of three anomaly detection algorithms in terms of their ability to detect indoor temperature anomalies is compared. Based on the fault tree analysis (FTA), a decision-making tree is developed to assist facility management (FM) professionals in identifying corresponding failed assets according to the detected anomalous symptoms. The AR system facilitates easy maintenance by highlighting the failed assets hidden behind walls/ceilings on site to the maintenance personnel. The system can thus provide enhanced support to facility managers in their daily O\&M activities such as inspection, recording, communication and verification.

\section{Findings:}

Taking the indoor temperature inspection as an example, the case study demonstrates that the O\&M management process can be improved using the proposed AR enhanced inspection system. Comparative analysis of different anomaly detection algorithms reveal that the binary segmentation-based change point detection is effective and efficient in identifying temperature anomalies. The decision-making tree supported by FTA helps formalise the linkage between temperature issues and the corresponding failed assets. Finally, the ARbased model enhanced the maintenance process by visualising and highlighting the hidden failed assets to the maintenance personnel on site.

\section{Originality/value}

The originality lies in bringing together the advances in augmented reality, digital twins and data-driven decision-making to support the daily O\&M management activities. In particular, the paper presents a novel binary segmentation-based change point detection for identifying temperature anomalous symptoms, a decision-making tree for matching the symptoms to the failed assets, and an AR system for visualizing those assets with related information.

Keywords: Digital Twin, Anomaly Detection, Augmented Reality, Operations and Maintenance

\section{Introduction}

It is undeniable that the tech-savviness is spreading to the building industry, which accelerates the adoption of digital strategy and data science in this area. Getting away from the old-fashioned "brick and mortar" way of working, digitalisation is reinventing the way we plan, deliver, operate, maintain and manage our infrastructure. A significant amount of data, generated throughout the digitalisation, carries great value. Artificial intelligence and machine learning (AI/ML) are just the keys to unlock this value. In this context, this paper examines how digital transformation in the buildings sector can generate value to both the building owners as well as users through effective operation and maintenance (O\&M) management.

Building Information Models (BIM) can be used as a data repository and an information source for supporting daily O\&M activities in buildings (Lu et al. 2018). In fact, BIM has been shown to reduce the time taken to update databases in the O\&M phase by $98 \%$ (Ding et 
al. 2009). BIM does not however provide a complete solution for whole-life asset management since the key focus of BIM is to serve as a digital representation of buildings and the assets within them. From the perspective of information richness and analytical/decision-making capability, the concept of Digital Twins (DT) is broader than BIM. In addition to the capabilities offered by BIM, DT is a comprehensive solution that can monitor the as-is status (e.g., condition) of its 'physical twin' by integrating multi-source data and data analytics, control and simulation functions (Eastman et al. 2011; Li et al. 2017). Effective decision-making to manage complex building systems requires integration and analysis of data from multiple sources. There is still a need to establish a unified data management platform so that heterogeneous data can be integrated safely and securely, and the approaches and technologies used must ensure effectiveness and efficiency when implemented in O\&M phase. Research related to the use of digital twins in building O\&M management is still at a very early stage, but increasingly gaining popularity (Lu et al. 2020a; Pishdad-Bozorgi et al. 2018; Shou et al, 2020).

In the O\&M phases, monitoring building environmental condition (e.g., temperature, humidity and $\mathrm{CO}_{2}$ concentration) has been a topic of great interest in the past few decades. Satisfactory indoor environment contributes not only to occupant well-being and productivity, but also reduces operational carbon associated with the provision of thermal comfort (Vellei et al. 2017). In this context, it is estimated that one in five buildings will become smart buildings by 2020 , containing over 50 billion Internet of Things (IoT) devices (Memoori 2014). The accurate and timely information provided by such technologies is capable of aiding facility managers in the process of detecting environmental anomalies and making corresponding decisions on operation and maintenance.

The long-term value of DTs and their integration with environmental anomaly detection techniques can be enhanced with the implementation of augmented reality (AR) or other interactive technologies (Gao and Pishdad-Bozorgi 2019; Wang et al. 2020). Indeed, the development of a visualised inspection system enhanced with AR opens enormous potential for the improvement in O\&M fieldwork from the perspective of easily accessible information support. Here, the AR-enhanced visual inspection system allows the cognitive connection to various types of O\&M information, such as building geometries and monitored indoor environmental conditions.

Taking temperature as an example of indoor environmental indicator, this paper seeks to address the challenge facility managers face in automatically detecting and interpreting temperature anomalies of building spaces. In particular, this paper explores how DTs and environmental anomaly detection techniques can improve the efficiency of inspection management when integrated with augmented reality (AR) solutions.

\section{Literature Review}

2.1 Building inspection system development

There is a growing consensus nowadays about the need to maximize the value of assets, and effective inspection of the as-is conditions is a key driver to achieve this (NIC, 2017). A great 
effort has been put into enhancing building inspection knowledge and inspection system development to assist facility management (FM) professionals in their decision-making processes (see Table 1). Previous research has focused on facilitating O\&M inspection via the FM information systems (e.g. Computerized Maintenance Management Systems, Computerized Aided Facility Management Systems) and sensor systems (Ferraz et al. 2016). However, the maintenance records within most of these systems only provide brief descriptions about repair activities (such as time, cost, and location), and are prone to missing or wrong records (Chang and Tsai, 2013). FM professionals are responsible for inspection scheduling and maintenance planning, such as when dealing with structural health problems or air-handling unit failures (Yu et al. 2014), to optimize the building performance. The absence of a logical framework to classify a building's pathological situations may result in misleading the decisions of FM professionals and site workers. Therefore, a well-defined inspection and diagnosis system could improve both short-term and long-term performance of the existing buildings.

BIM, cloud storage and cloud computing technologies are being adopted by industry to form project-based collaborative platforms for improving the O\&M management quality and customer experience (Zhan et al. 2019). A decision support system (DSS) that analyses the data collected from sensors, merges them with engineering knowledge about the system, and turns this into a visual maintenance/ inspection task execution system is indeed a need of the hour (Agnisarman et al. 2019. Importantly, such a system should be capable of supporting maintenance and inspection site workers with useful information regarding assets that are often hidden from sight (e.g., inside the walls/ceiling structures).

Table 1. A brief summary of inspection system development

\begin{tabular}{lll}
\hline Author/year & Key technologies/algorithm & Key contribution \\
\hline $\begin{array}{l}\text { Guo and } \\
\text { Wang (2013) }\end{array}$ & $\begin{array}{l}\text { Fault diagnosis expert system } \\
\text { incorporating association rule and } \\
\text { data analysis }\end{array}$ & $\begin{array}{l}\text { Implementing fault diagnosis in } \\
\text { the Electrical Multiple Unit }\end{array}$ \\
\hline $\begin{array}{l}\text { Memarzadeh } \\
\text { and Pozzi }\end{array}$ & $\begin{array}{l}\text { Partially Observable Markov } \\
\text { Decision Process (POMDP) } \\
\text { considering Value of Information } \\
\text { (VoI) }\end{array}$ & $\begin{array}{l}\text { Integrating inspection } \\
\text { scheduling and maintenance } \\
\text { planning for infrastructure } \\
\text { systems }\end{array}$ \\
$\begin{array}{l}\text { de Angelis et } \\
\text { al. (2016) }\end{array}$ & $\begin{array}{l}\text { Monte Carlo strategy using efficient } \\
\text { general numerical technique }\end{array}$ & $\begin{array}{l}\text { Providing a solution which } \\
\text { trades off between the costs } \\
\text { associated with multiple } \\
\text { inspection and repair activities }\end{array}$ \\
\hline $\begin{array}{l}\text { Au-Yong et } \\
\text { al. (2017) }\end{array}$ & $\begin{array}{l}\text { Descriptive analysis, correlation } \\
\text { analysis and regression with data } \\
\text { collected from questionnaire and } \\
\text { interviewing }\end{array}$ & $\begin{array}{l}\text { Investigating the association } \\
\text { between system breakdown rate } \\
\text { and frequency of inspection }\end{array}$ \\
$\begin{array}{l}\text { Hamledari et } \\
\text { al. (2017) }\end{array}$ & $\begin{array}{l}\text { Developed algorithm updating } \\
\text { BIMs (Industry foundation classes } \\
\text { IFC schema) based on user criteria }\end{array}$ & $\begin{array}{l}\text { Automating site-to-BIM data } \\
\text { transfer and supporting reality- } \\
\text { capture techniques }\end{array}$ \\
\hline
\end{tabular}




\begin{tabular}{lll}
\hline & and analyzing data & \\
\hline $\begin{array}{l}\text { Schneider et } \\
\text { al. (2018) }\end{array}$ & $\begin{array}{l}\text { Heuristics method to define } \\
\text { inspection and repair strategies }\end{array}$ & $\begin{array}{l}\text { Identifying optimal inspection } \\
\text { and repair strategies for offshore } \\
\text { jacket structures }\end{array}$ \\
\hline $\begin{array}{l}\text { Bortolini and } \\
\text { Forcada } \\
(2018)\end{array}$ & $\begin{array}{l}\text { Asset analysis, operation analysis, } \\
\text { and risk analysis using online } \\
\text { survey }\end{array}$ & $\begin{array}{l}\text { Evaluating the technical } \\
\text { performance of existing } \\
\text { buildings }\end{array}$ \\
$\begin{array}{l}\text { Zhan et al. } \\
(2019)\end{array}$ & $\begin{array}{l}\text { Image classification algorithms } \\
\text { supported by BIM knowledge } \\
\text { repository }\end{array}$ & $\begin{array}{l}\text { Improving and automating the } \\
\text { inspection-repair processes }\end{array}$ \\
\hline
\end{tabular}

2.2 Inspection for indoor environmental anomalies

Currently, a large portion of modern building management systems (BMSs) or building automation systems (BASs) are pre-programed to trigger alarm notifications for apparent anomalies from an energy perspective (e.g. HVAC energy consumption) and an indoor environmental perspective (e.g. room temperature and humidity). Due to the fact that environmental data collected by many standard building management systems are often inaccessible and available for analysis, wireless sensors are proven to be a popular alternative for efficiently collecting such data (Wu and Clements-Croome 2007), especially considering the emergence of advanced sensing techniques such as Micro Electro Mechanical Systems (MEMS) (Hautefeuille et al. 2008). In new buildings, WSN can be implemented easily with low-cost low-power wireless sensor nodes, and existing buildings with WSNs can be upgraded simply by adding extra sensor nodes. BMS or WSN can provide rich raw data about the monitored environment, and the critical next step is to analyse such data to identify anomalous behaviour. Instead of manually inspecting the anomalies through control-charts or trend analysis, energy or environmental performance anomaly detection is usually achieved in BMSs using simple rule-based methods. The rules adopted are generally acquired from experienced facility managers based on the design parameters of building systems. For instance, if the room temperature goes outside a predefined range, an alarm would be triggered by BMS for the attention of facility managers. Alternatively, rules can also be learned through a data mining-based decision support system (Pena et al. 2016), which also requires extensive expert engagement. However, in practice, facility managers usually operate with very constrained resources and are typically overburdened by excessive number of alarms caused by these rules.

In response to this situation, a number of automated supervised or unsupervised data analytics techniques supported by machine learning are emerging to aid facility managers in identifying valid anomalies without explicitly modelling the thermal properties and behaviour of buildings (Horrigan et al. 2018). Numerous supervised machine learning techniques, such as linear regression (Korolija et al. 2013), support vector machine (Li et al. 2009) or neural network (Mustafaraj et al. 2011), are available to predict building performance trained on a set of selected historical building data. Supervised analytics are powerful in modelling complicated relationships within operational data. However, these methods need to be trained 
and tested on extensive quantities of historical data under exhaustive operational scenarios, which is often not available.

Unsupervised techniques, on the other hand, are more promising in discovering potentially useful yet previously unknown intrinsic correlations and patterns in data given limited prior knowledge (Fan et al. 2018). Unsupervised anomaly detection techniques can be classified into three classes: nearest neighbour based, clustering based, and statistical methods.

Wijayasekara et al. (2014) utilized a modified nearest neighbour clustering algorithm and a fuzzy logic rule extraction technique to establish a model of normal building energy behaviour and identify anomalies that do not conform to the model. Capozzoli et al. (2015) compared the performance of K-Means, Density-Based Spatial Clustering of Applications with Noise (DBSCAN), artificial neural networks and other algorithms on detecting anomalous energy consumption patterns. The results indicated that DBSCAN is capable for identifying anomalies in multivariate data, which is further verified by Jalori and Reddy (2015).

Statistical methods, on the other hand, are found to be the widely accepted tool used for detecting anomalies. Statistical process control (SPC) techniques, one of the most widely used statistical methods across many industrial applications, have also been used to identify building performance problems (Sun et al. 2013). Essentially, SPC techniques, such as Shewhart, cumulative sum (CUSUM) and exponentially weighted moving average (EWMA) control charts, are unsupervised approaches that can recognize statistically meaningful performance problems according to the data consistency rather than knowledge learned from anomaly labelling. Barbeito et al. (2017) demonstrated the application of a residual based Shewhart chart to the energy dataset in a web platform, where the occurrences and causes anomalies in HVAC systems were detected. Wang and Chen (2016) presented the design of a residual-based EWMA control chart used for detecting anomalies of air handling units, in tandem with an expert rule system. These studies showed that CUSUM and EWMA based monitoring are generally more sensitive in detecting small or gradual shifts in the process. Another statistical analytical technique to reveal unintended shifts in building performance is change point detection. This aims to statistically discover time points at which the behaviour of time series building performance changes in an unsupervised manner. Pereira and Ramos (2018) proposed a new method using change point analysis of in-situ time series of environmental parameters to detect occupants' activities of daily living (ADLs) in residential buildings. Li et al. (2019) recognized the energy consumption patterns in residential buildings using an available binary segmentation method in ' $R$ ' programming software.

However, most of the studies so far focused on analysing the building energy behavioural patterns and identifying emerging energy consumption related anomalies. Detection of environmental anomalies, while significantly influencing occupants' wellbeing and productivity, has not been studied adequately. Bosman et al. (2013) proposed to use a lightweight machine learning technique to reveal anomalous issues in sensor data produced by long-lived deployed sensors, based on supervised Linear Least Squares Estimation (LLSE). Bosman et al. (2015) developed a heterogeneous set of local online learning 
classifiers, each performing an incremental unsupervised learning on recognizing anomalies. Labelled datasets are essential in evaluating the performance of these methods, while in realworld datasets the labelling is not always exact and largely depends on expert knowledge. This study proved its effectiveness in identifying indoor environmental events, based on its statistical properties and the cross-referencing of building asset information.

However, the choice of an appropriate technique is not straightforward. This paper seeks to present effective and efficient methods that identify building temperature anomalies based on limited available building time-series data, in order to develop an inspection decision support system.

\section{Methodology}

The proposed AR-enhanced inspection system (as shown in Fig.1), is incorporated into the general process of daily O\&M management. Various non-geometric O\&M data sources and BIM are integrated through the building DT platform. The DT analyses the as-is condition of the environment using anomaly detection functions and is able to extract data regarding related assets from other integrated data sources by querying through the BIM model. Meanwhile, the professional O\&M knowledge (e.g. fault trees and cause-effect relationships) is conveyed through knowledge representation models. The FM professionals can be kept informed of the analysis results through the DT platform and the corresponding tasks can be assigned to the site workers accordingly. Additionally, the visualization enhanced inspection tool (i.e. AR equipment) with embedded information display modules can be launched to provide visualisation of hidden assets to maintenance personnel.

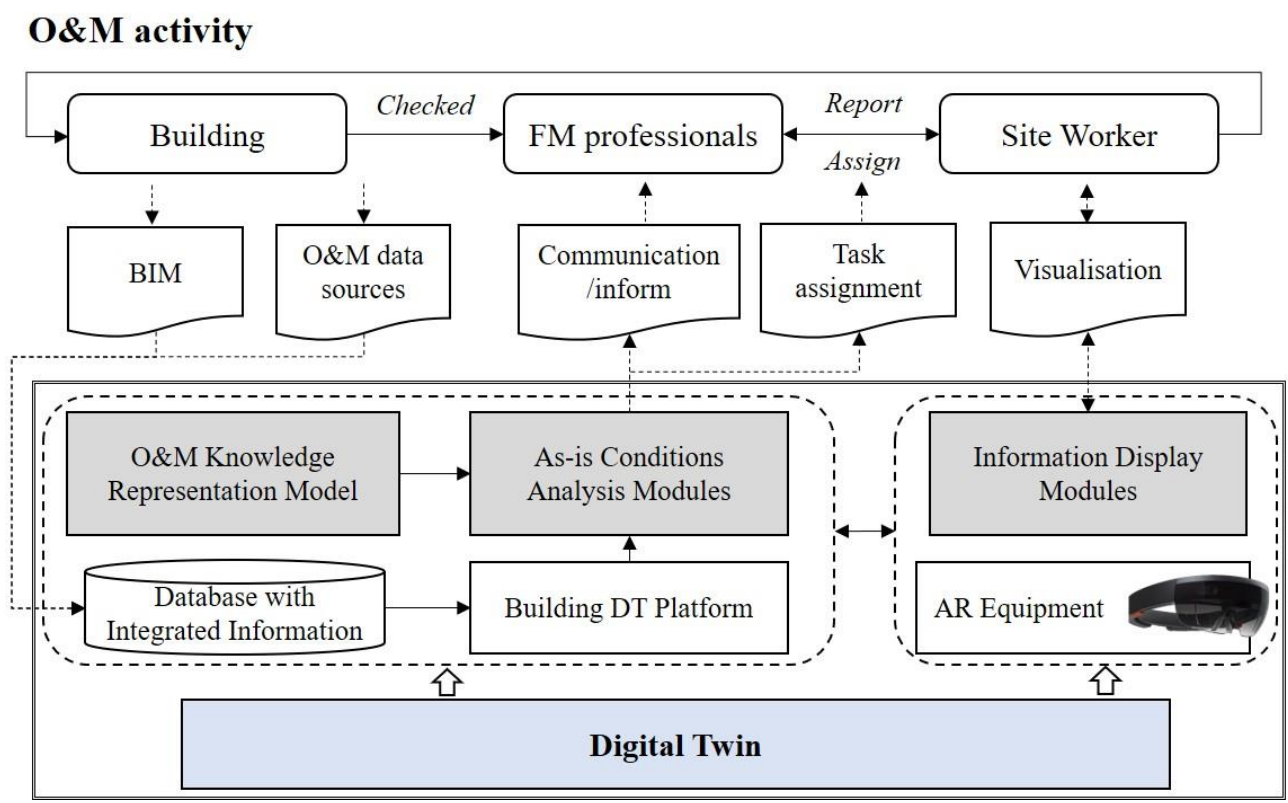

Figure 1 . The proposed process of the visualised inspection system in daily O\&M management 


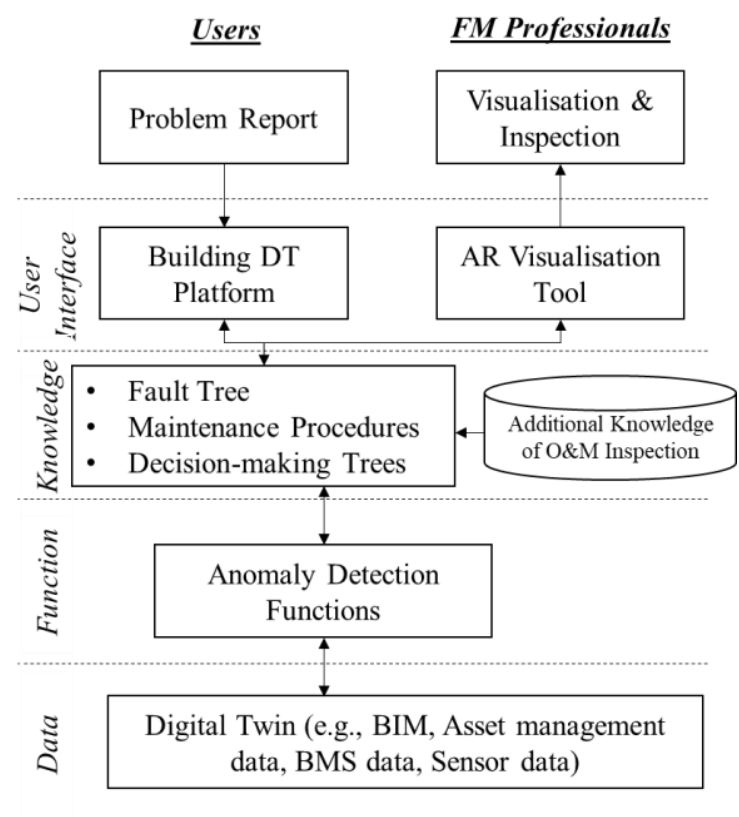

Figure 2. The architecture of the proposed inspection system

As shown in Fig. 2, the architecture of the visual inspection system consists of four layers. It was developed based on the concepts presented in Motamedi et al. (2014). Firstly, a building DT platform in the data layer is established as the data platform that can integrate data from different sources, respond to data queries and further store information feedback collected from site workers/FM professionals.

In the function layer, typical anomaly detection algorithms are implemented to analyse the condition of the indoor environment. Three types of statistical methods: moving average (MA), cumulative sum (CUSUM) and a binary segmentation-based change point detection method, are implemented for detecting anomalies in the environmental data.

Starting from a naive hypothesis, that is "tomorrow is going to be the same as today", the moving average of the previous day ( $k$ values) is considered to be the expected value of the present day: $\tilde{y}_{t}=\frac{1}{k} \sum_{i=1}^{k} y_{t-i}$. The anomalous alarm is triggered once the observed value exceeds the predefined confidence band.

The CUSUM algorithm, alternatively, detects time instants of change by sequentially accumulating deviations from the anticipated value, and alarms when the tracking statistics exceed the control limits. The two-sided CUSUM version (De Oca et al. 2010) designed for non-stationary data is based on calculating the following two tracking statistics:

$S_{i}^{+}=\max \left\{0, S_{i-1}^{+}+y_{i}-Q_{\tau_{i}}(\alpha)\right\}, S_{i}^{-}=\max \left\{0, S_{i-1}^{-}+Q_{\tau_{i}}(1-\alpha)-y_{i}\right\}$, where $Q_{\tau_{i}}(\alpha)$ and $Q_{\tau_{i}}(1-\alpha)$ denote the upper and lower $\alpha$-percentiles respectively, during the screened sustained period of historical data. 
The binary segmentation-based change point detection algorithm (Fryzlewicz 2014) is another promising candidate in statistically separating normal and anomalous time periods. The binary segmentation is an iterative process. A single change point test statistic is first applied to the entire dataset and the dataset is split into two at the found change point location by minimizing the objective function $\underset{1 \leq \tau_{1}<\cdots<\tau_{m} \leq n-1}{\arg \min } \sum_{i=1}^{m+1}\left[C\left(y_{\tau_{i-1}+1: \tau_{i}}\right)+\beta\right]$, where $\tau_{i}$ is the $i^{\text {th }}$ change point; $C(\cdot)$ denotes the cost function incurred from a segment of the data, can be either quadratic error loss, Huber loss, or the negative log-likelihood; and $\beta$ is the penalty when introducing a change point, which avoids overfitting. The procedure is repeated at the two separated datasets until no change point is found in any part of the dataset. An appropriate algorithm that shows better performance in identifying environmental anomalies is identified through comparative analysis and preliminary tests in the studied case.

In the knowledge layer, a comprehensive classification and categorisation of temperature issues relating to building assets is constructed based on fault tree analysis (FTA). This encompasses diverse events to distinguish different kinds of commonly appearing temperature issues in daily O\&M management. FTA is a graphical tool for analysing possible causes of a failure (Yu et al. 2014, Motamedi et al. 2014). It can be used to model the logical relationships and further evaluate different events qualitatively and quantitatively. Corresponding to different events, common failure sub-categories and associated assets are identified. Following this, a decision-making tree was designed and developed to match between the anomalous temperature events with the corresponding failed assets. The knowledge layer is essential for the semi-automation of the inspection process. The expert knowledge conveyed through the classification and categorisation using FTA enables the event-asset matching, which is conducted manually in this paper but is possible to be automated using machine learning techniques once sufficient data through the human-driven process is gathered.

The traditional practice of inspection is to have an index sheet and relevant drawings at the work sites, which is usually a time-consuming, tedious, and error-prone process, especially when checking specific details. An AR tool is introduced for improving human perception and cognition with both real and virtual information sources (Hou et al. 2014). In the user interface layer, an AR model and a DT platform is created and incorporated to assist inspection and identification of faulty assets. The user interface layer has two main functions: a) to link the identified temperature issues with their corresponding assets; and b) to guide site workers in finding the target assets behind decorations using the AR equipment.

\section{Case Study Development and Evaluation}

This system was deployed at the Institute for Manufacturing (IfM) building at the University of Cambridge. The IfM building is a three-story building at the West Cambridge site and includes study, office, research and laboratory spaces, each with different assets such as a radiator, air handling units and a variable refrigerant flow (VRF). 


\subsection{Data layer (Digital Twin establishment)}

Referring to Fig.3, this dynamic DT platform was developed based on the system architecture proposed by Lu et al., 2019. This developed DT was integrated with the data acquired from the building management system (BMS) in addition to Monnit wireless IoT sensors that were used to gather temperature and humidity data with a one-minute heartbeat. The sensor data as well as the data from the BMS was uploaded to the DynamoDB NoSQL database provided by Amazon Web Services (AWS) using the Hyper Text Transfer Protocol (HTTP). Further information about the setup of the building digital twin can be found in Lu et al. (2020b).

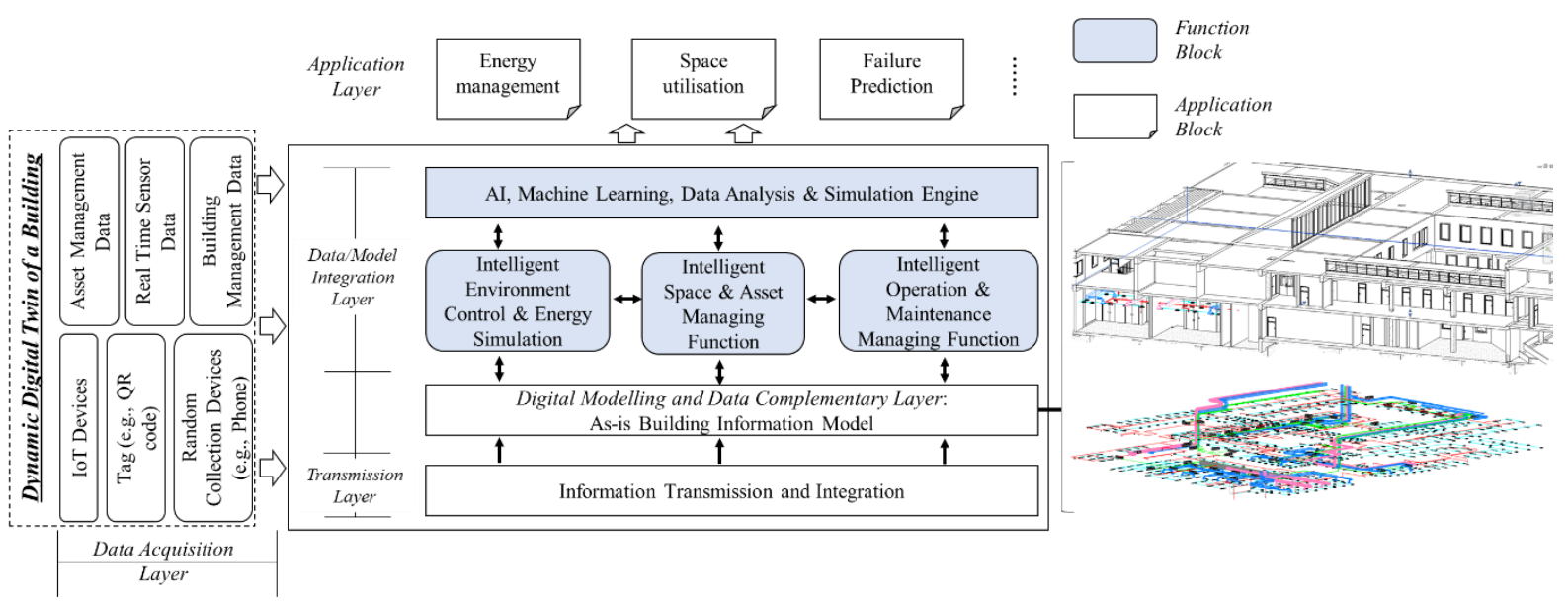

Figure 3. The system architecture of the building DT (modified according to Lu et al. 2019)

\subsection{Function Layer}

On the basis of the temperature data collected by the sensors, anomalous behaviour of the indoor environment is detected to provide important clues for the diagnosis of the HVAC system. Fig. 4 shows the temperature log obtained from a seminar room in the building during the period from $1^{\text {st }}$ July 2018 to $1^{\text {st }}$ October 2018. According to the mechanical, electrical and pumping drawings of the building, the seminar room is conditioned by a VRF air conditioning system, in which the water pump plays a significant role. Pump maintenance records were obtained from the facilities management system, which indicated that a pump malfunction event occurred between $19^{\text {th }}$ July and $8^{\text {th }}$ August (period of interest).

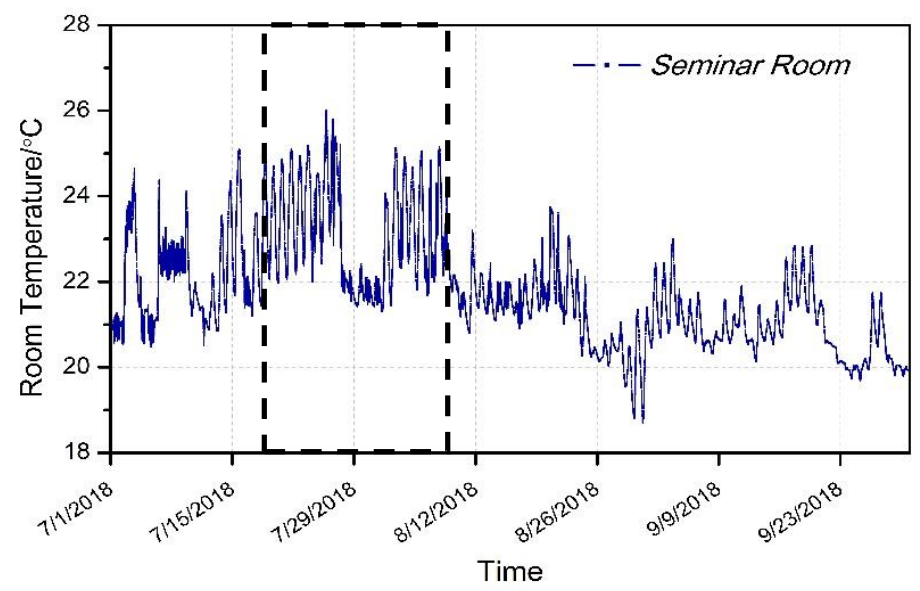

Figure 4. Temperature logs in seminar room of IfM 
The performance of three anomaly detection algorithms, including moving average (MA), cumulative sum (CUSUM) and a binary segmentation-based change point detection method, were evaluated to examine their ability to detect the aforementioned anomaly.

Moving average is a basic and easy to execute method. Here, the moving average temperature on the daily basis (past 24 hours, $k=24$ ) is taken as the expected temperature of the present moment. An alarm is triggered once the observed value exceeds the predefined confidence band. As shown in Fig. 5, applying daily smoothing on past data allows us to clearly observe the dynamics of temperature. MA is found to be capable of detecting several anomalies observed during our period of interest. However, various anomalies that sit outside the period (false positives) are also evident, which would be troublesome to facility managers.

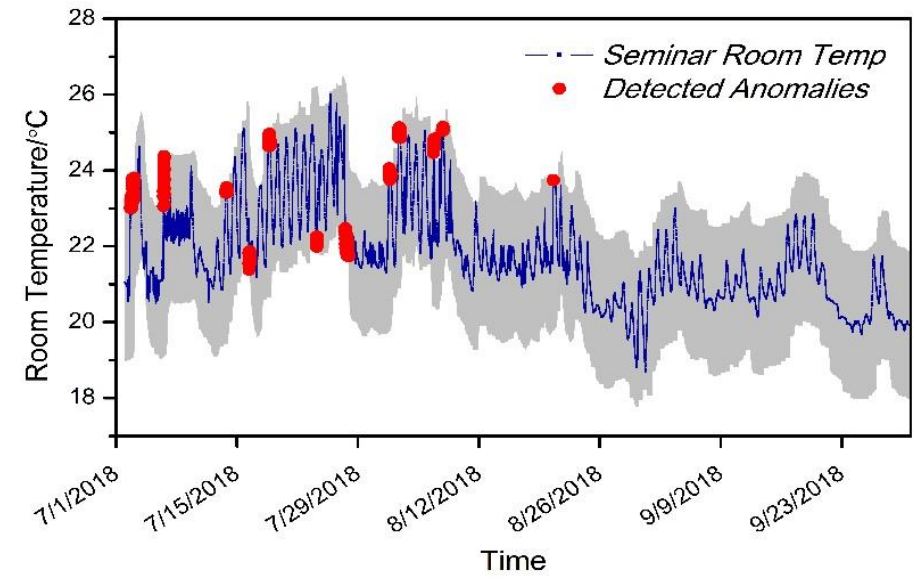

Figure 5. Anomaly detection results of moving average (MA)

From the perspective of decreasing the false positive rate (i.e., the fraction of observed non-anomalies that are false positive events), CUSUM is adopted to reveal anomalous temperature issues during the same period of pump malfunction. The CUSUM algorithm calculates the deviations of monitored temperature (relative to target means) in an accumulative way and rejects the null hypothesis of no change-point when the two tracking statistics $S_{i}^{+}$or $S_{i}^{-}$gets too large. Fig. 6 demonstrates the detection results of the CUSUM control chart. Before the facility manager reports the malfunction $\left(19^{\text {th }} \mathrm{July}\right)$, CUSUM timely detects a statistically meaningful change point. After the pump replacement ( $8^{\text {th }}$ August), the environmental performance gradually gets back to normal. Compared with MA, CUSUM could better identify the anomalous temperature issues, which contributes to tracing back to the failure or malfunction of relevant building assets. 


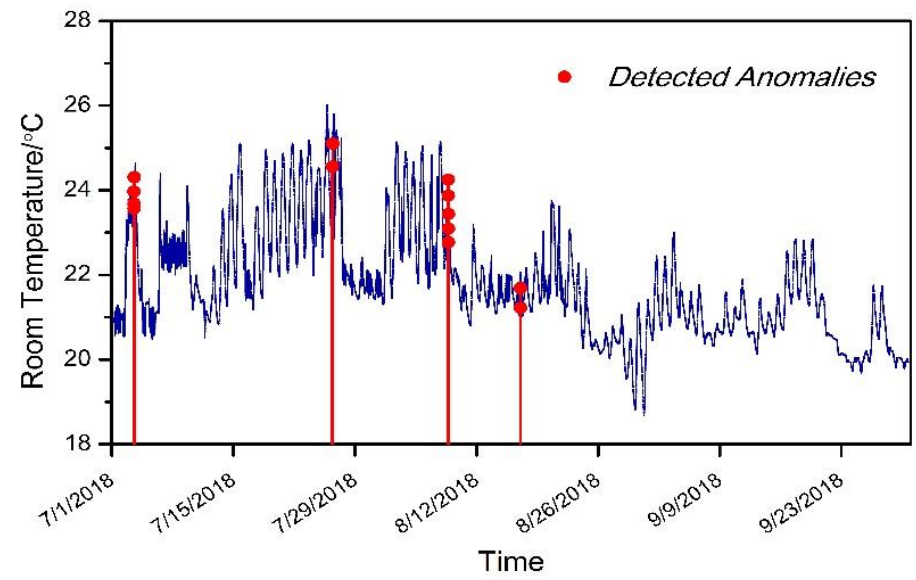

Figure 6. Anomaly detection results of cumulative sum (CUSUM) control chart

Instinctively, if the CUSUM chart gives an out-of-order indication in spatial temperature, then it is reasonable to suspect that there is an anomaly somewhere before the identified change point. Indeed, the CUSUM algorithm is particularly effective in detecting abrupt changes in time series, but without specifically indicating the severity level of these changes. CUSUM is generally unable to identify the duration of anomalies, as its primary objective is to point out the moments when the monitored temperature deviates from its previous behaviour.

In order to address the drawbacks of CUSUM, a binary segmentation-based change point detection is applied to identify the critical change period in the data, by highlighting its starting and end time respectively, as shown in Fig. 7. The x-coordinates of the vertical lines indicate the detected change point locations. The five estimated change point locations are mostly distributed around the anomalies found by CUSUM. Binary segmentation provides clearer boundaries between periods with different statistical properties $\left(14^{\text {th }}-27^{\text {th }} \mathrm{July}, 27^{\text {th }}\right.$ July- $1^{\text {st }}$ Aug and $1^{\text {st }}-9^{\text {th }}$ Aug). The impact of pump malfunction event occurring during the period of interest is clearly identified. In addition, binary segmentation is an approximation algorithm with the computational complexity of $O(m \log m)$. Thus, it only requires lightweight computing power, which is suitable for continuous anomaly detection.

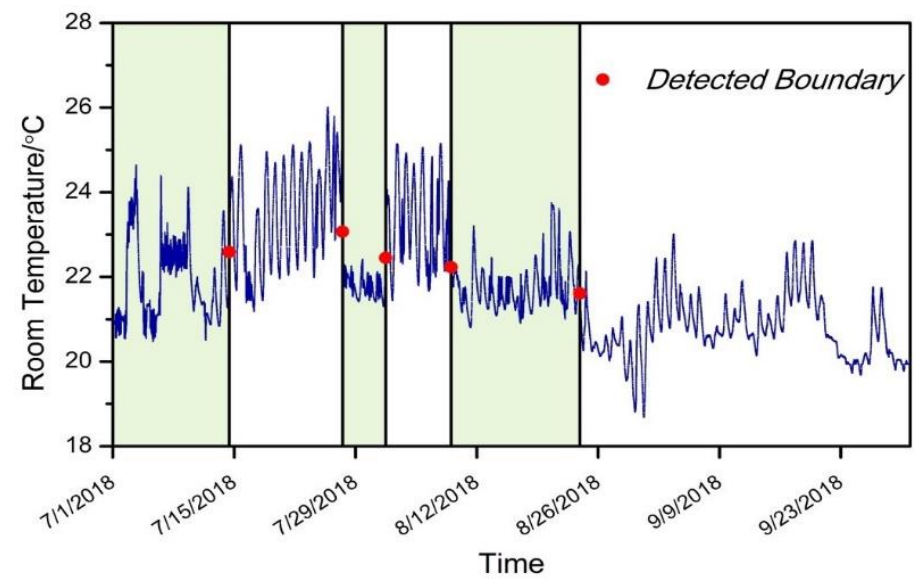

Figure 7. Anomaly detection results of binary segmentation-based change point detection 


\subsection{Knowledge Layer}

In the knowledge layer, temperature issues detected via the function layer are assessed to ascertain whether it is an asset-failure related problem (see Fig. 8). If it is, the space ID and the target asset ID of failed assets are provided through the building DT platform (i.e., DT is linked with the BMS database).

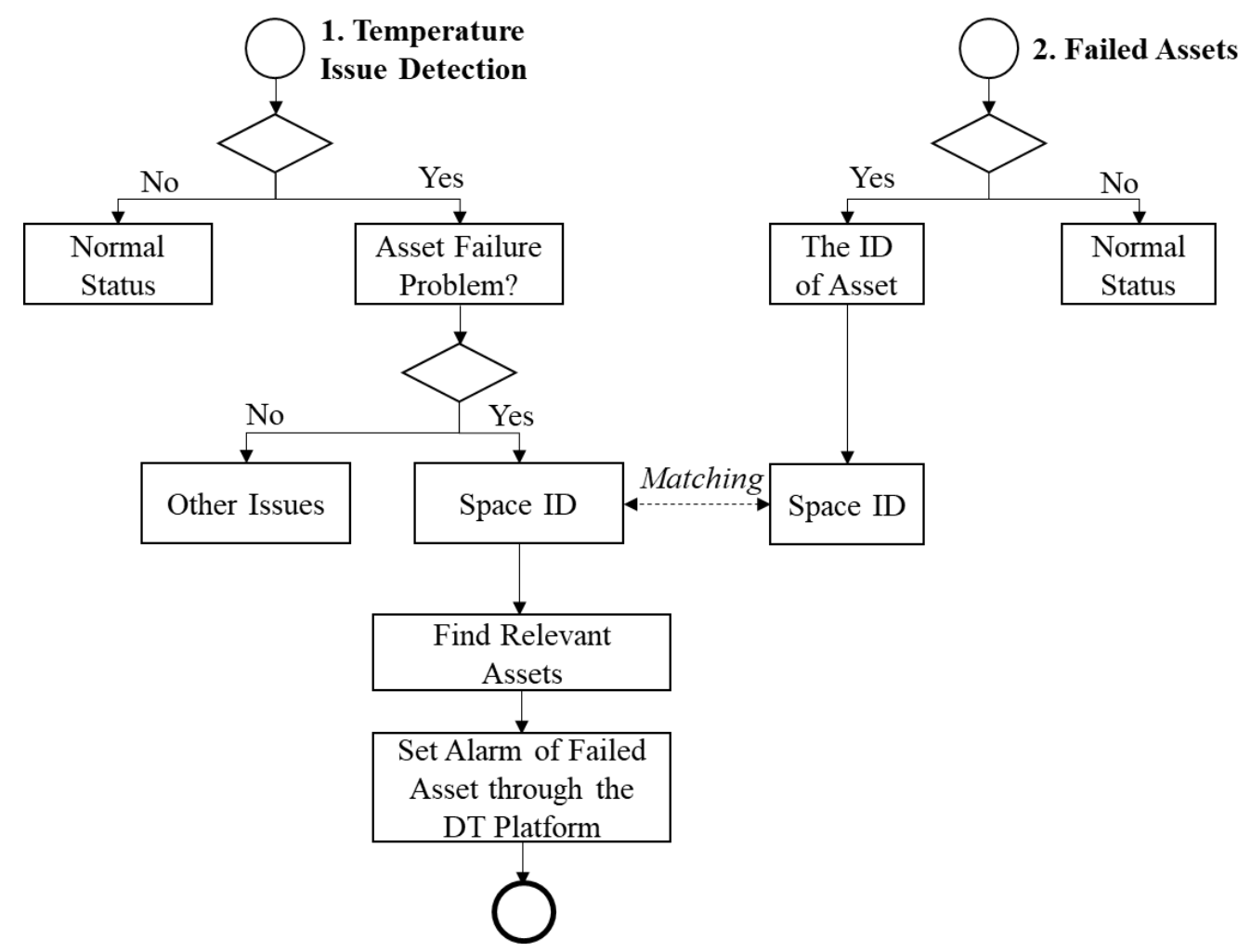

Figure 8. The decision-making tree of making decisions and matching with relevant failed assets

Within the building DT, the corresponding assets have been classified and allocated for each space. By matching the assets with each space ID, the failed assets (including locations) can be confirmed and presented through the building DT platform. Based on the detected failures for each space, the 'real-time' status of each asset can be ascertained from the developed DT, which could help facility managers to verify the failed assets based on the FTA. A list of analysed temperature issues relating to building assets using FTA can be seen in Fig.9, which is summarised through the interview with FM professionals. 


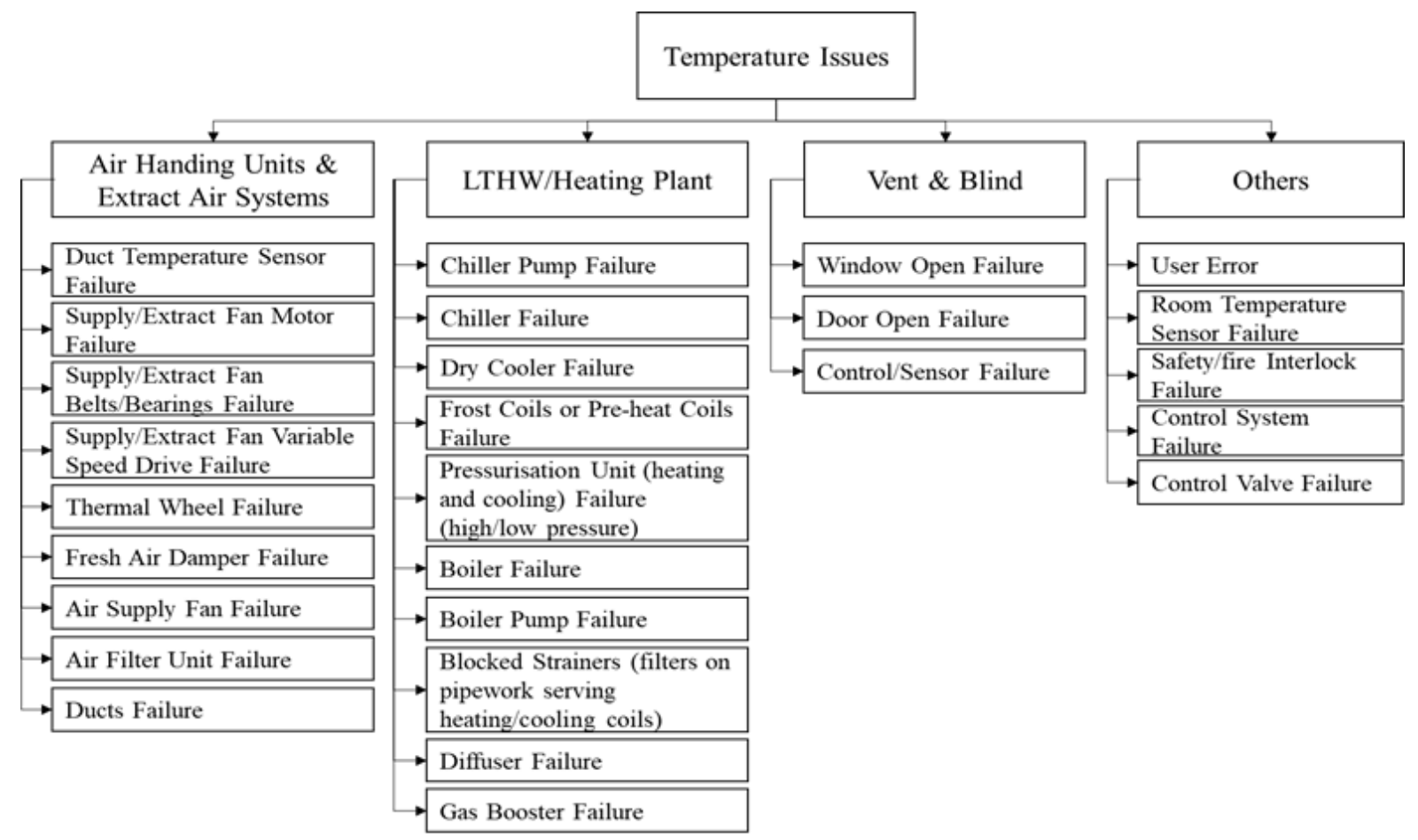

Figure 9. Fault tree analysis of temperature related issues for the IfM building

\subsection{User Interface Layer}

Based on the designed processes and equipment, a DT user interface platform and an AR model have been developed for communicating between FM professionals and site workers (see Fig.10). In this study, the seminar room was used as an example. The associated data was integrated and analysed and the inspected as-is condition was demonstrated using the DT user interface. Microsoft HoloLens 1 was used in this project for presenting the AR models. The working processes are presented in Fig.11. If there are temperature anomalies in the seminar room identified by the function layer, the FM professionals would discover the alarm from the DT platform and gain 'real-time' information about the failed assets. Thus, based on provided information from DTs, site workers could use AR techniques and find the target assets in the corresponding spaces. 

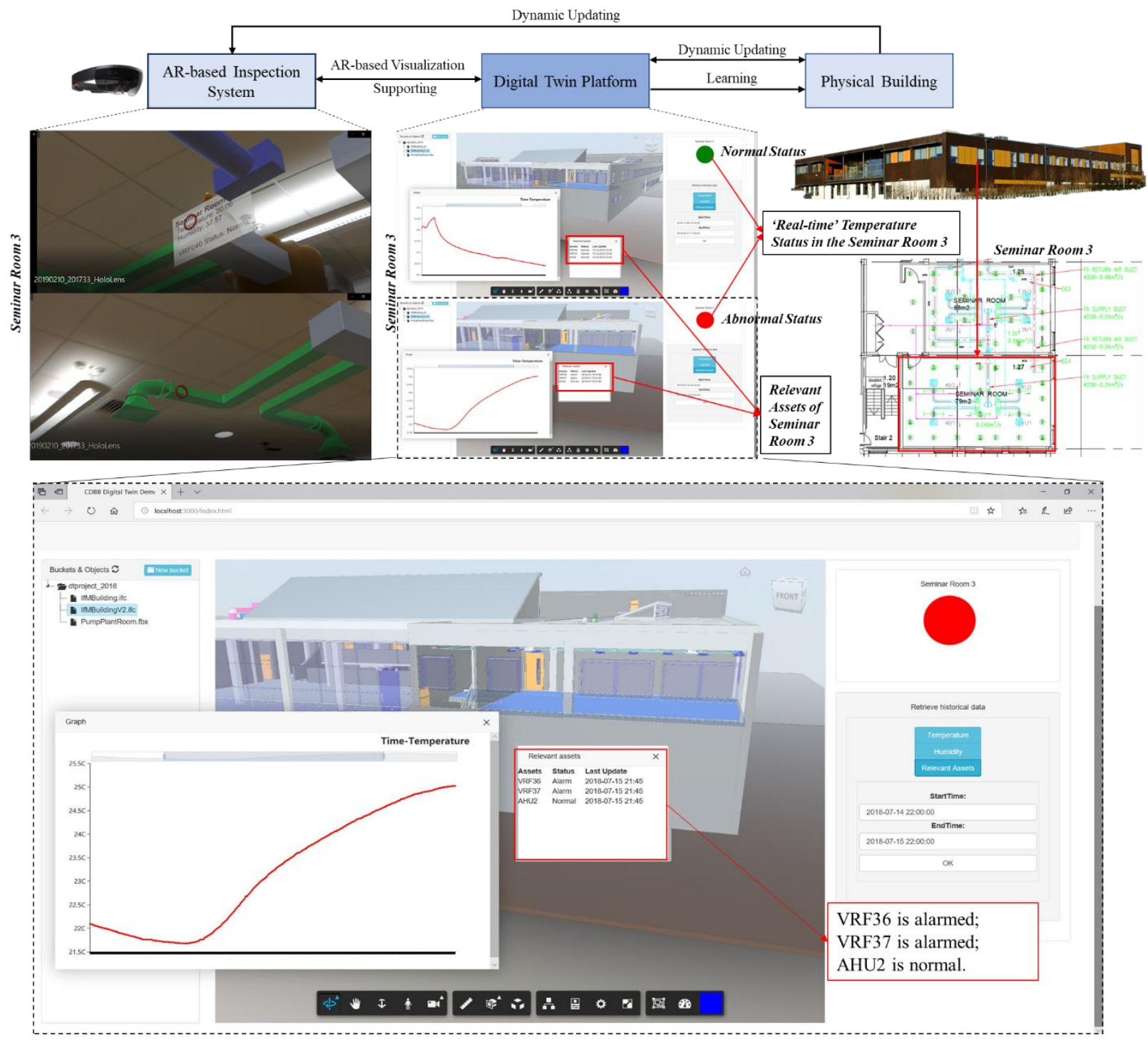

Figure 10. The DT user interface and the launched AR model

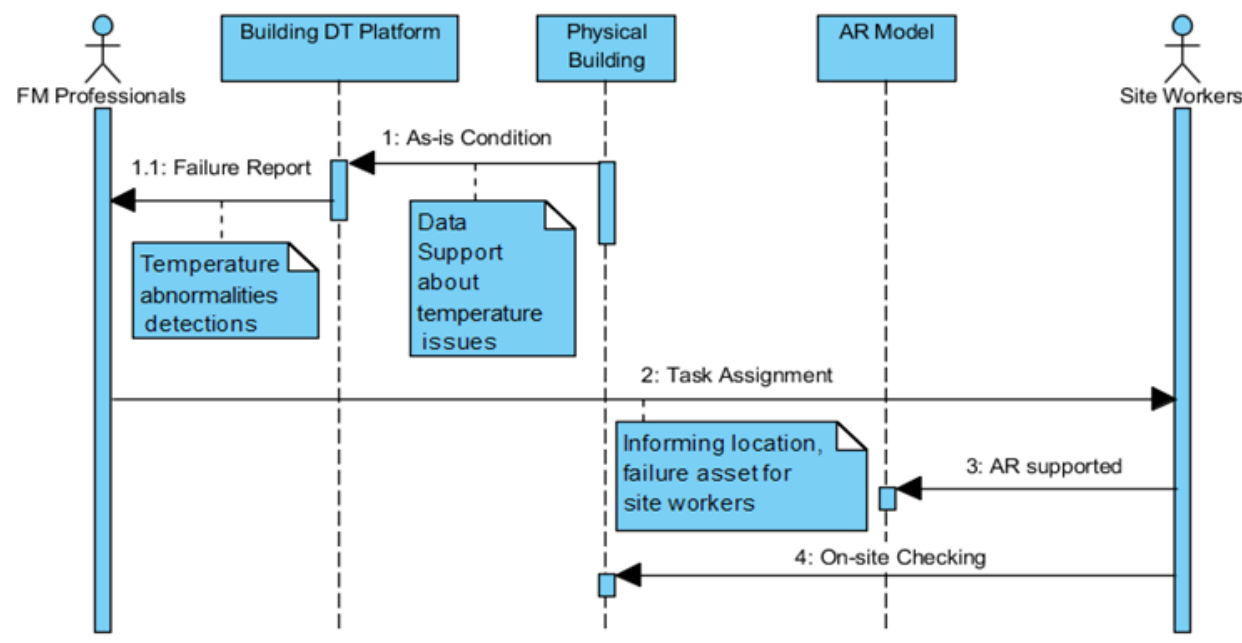

Figure 11. The inspection working process expressed by UML diagram 


\section{Discussion}

\subsection{Summary}

A comfortable indoor environment has a great influence on the productivity and well-being of human occupants. With the trend of digitalisation of the built environment, automated inspection for the indoor environment without excessive intervention from facility managers is becoming increasingly possible. Essentially, decisions regarding the occurrence of environmental anomalies are usually made based on various types of accumulated historical data collected using sensors and the BMS. It must be pointed out that DT with a BIM embedded plays a significant role in facilitating the availability and accessibility of heterogeneous environmental parameters, which enables data from different stakeholders in the O\&M phase of a building to be created, shared, exchanged and managed. Instead of requiring extremely busy facility managers to subjectively evaluate the environmental condition, data analytics techniques are shown to enable automatic extraction of anomalous environmental behaviour that do not conform to expectation.

For most current studies, one of the premises is that the targeted space environment has been monitored for a period so that sufficient data can be collected to train a model. In addition to the requirements of data volume, data under anomalous and non-anomalous scenarios needs to be manually labelled. The binary segmentation-based change point detection partially solves the problem of data scarcity by automatically partitioning the data according to its statistical properties. Due to the complexity of interrelations between different building components and systems, suspicious anomalies are not always caused by the failure or malfunction of building assets. Sending alerts regarding all the anomalous events to site workers and FM professionals is not a good and effective solution, because they cannot prioritise these anomalies according to their severity and possible consequence using only the detected anomalies themselves. The visualized inspection system presented in this paper shows the potential for integrating knowledge of deteriorated or failed assets (including their locations) and heuristically assisting FM professionals in filtering and finding the main cause of the major anomalies. Moreover, the visualized inspection system could also make it easier for the site workers to locate and repair failed assets when conducting maintenance tasks.

\subsection{Research Limitations}

It is infeasible to completely rely on building architectural information and first principles, because the gaps between as-built drawings and as-is condition can never be ignored.

Therefore, data driven methodologies are extensively used in daily O\&M management due to their broad applicability and simplicity in application. In this paper, anomaly detection for building environment is defined and implemented in function layer of building DTs, and the performances of different data-driven anomaly detection algorithms are evaluated. Although the proposed binary segmentation-based change point detection overcomes the requirement of large volume labelled data, still completely putting aside the physical information is not a good choice. Ideally, by combining physical prior knowledge with empirical data, the pattern 
behind the environmental parameters under normal conditions could be extracted, which provide a general metric classifying anomalous and non-anomalous data.

The building DT established in this case study was developed based on live data, which means that the dynamic DT is capable of tracing the as-is condition of assets and the indoor environment in a real time manner. The AR platform used by site workers was developed based on embedded databases, which include asset information and relevant historical data within a predefined set period of time. The failure assets can be found easily through this developed system, but cannot be highlighted automatically at this stage.

\subsection{Future work}

Instead of pure data-driven anomaly detection algorithms, data centric solutions that integrate knowledge from mathematical physics-based models are more likely to provide better decision making under enormously uncertainty. However, the synthesis of the mathematical model with the data is still unclear and represents a key challenge for statisticians and engineers. A possible way to synthesize is to estimate the unknown parameters in the physical model using observed data. Further improvement is needed to introduce prior knowledge into the O\&M management, including environmental anomaly detection.

Regarding the matching between found environmental anomalies and corresponding causes or failed assets, expert knowledge is still the dominant judgement criterion. But the knowledge is basically the understanding of symptoms for different asset failure scenarios. Summarizing the knowledge and realising automated matching between anomalous environmental symptoms and corresponding causes of asset failures is necessary.

In addition, in order to keep consistency with the developed dynamic building DTs, colour coding and real-time interaction functions in the AR platform will also be introduced in the future work. The colour coding will be used to highlight the failed assets on site and the real-time interaction functions (such as real-time queries) embedded in AR techniques will be used for presenting the as-is condition of assets.

\section{Conclusion}

In order to develop an easy-to-use and practical visualised inspection system for daily O\&M management, an inspection system based on building DTs was developed, specifically focused on spatial temperature anomalies. This was not only to demonstrate the application of the developed inspection system in detecting suspicious anomalies, but also to contribute to the advancement of research through the following:

- An architecture of DT-based visual inspection systems is proposed for daily O\&M, which is used to support identification of indoor environmental anomalies and corresponding failed assets;

- The binary segmentation-based change point detection is adopted to identify temperature anomalies indicated by change-points, without requiring large volume labelled data;

- A decision-making tree is developed to support matching between the found anomalous temperature symptoms and corresponding asset failures; 
- An AR based visualization tool is developed for site workers, which is capable of highlighting the failed assets with their related information.

The proposed system would provide a visualized way for site workers and FM professionals to detect temperature-relevant failures and develop solutions/plans for timely and effective inspection. Future work will be focused on improving the reasoning linkages between temperature anomalies and corresponding assets. In order to further improve this system, indoor localization of AR techniques for site workers will also be part of our future research directions.

\section{Acknowledgement}

This research that contributed to this paper was supported by the Centre for Digital Built Britain (CDBB) with funding provided through the Government's modern industrial strategy by Innovate UK, part of UK Research \& Innovation. It was also partly funded by the EPSRC/Innovate UK Centre for Smart Infrastructure and Construction (Grant Numbers EP/N021614/1 and 920035). This paper is based on a conference paper presented by the authors at the ASCE Construction Research Congress (CRC) 2020.

\section{Reference}

Au-Yong, C. P., Ali, A. S., Ahmad, F., and Chua, S. J. L. (2017). "Optimal inspection frequency to mitigate the risk of building system failure." Structural Engineering and Mechanics, 64(3), 347-352.

Agnisarman, S., Lopes, S., Madathil, K. C., Piratla, K., and Gramopadhye, A. (2019). “A survey of automation-enabled human-in-the-loop systems for infrastructure visual inspection." Automation in Construction, 97, 52-76.

Barbeito, I., Zaragoza, S., Tarrío-Saavedra, J. and Naya, S., (2017). "Assessing thermal comfort and energy efficiency in buildings by statistical quality control for autocorrelated data." Applied energy, 190,1-17.

Bortolini, R., and Forcada, N. (2018). "Building inspection system for evaluating the technical performance of existing buildings." Journal of Performance of Constructed Facilities, 32(5), 04018073.

Bosman, H.H., Liotta, A., Iacca, G. and Wörtche, H.J., 2013, October. Anomaly detection in sensor systems using lightweight machine learning. In 2013 IEEE International Conference on Systems, Man, and Cybernetics (pp. 7-13). IEEE.

Bosman, H.H., Iacca, G., Tejada, A., Wörtche, H.J. and Liotta, A., (2015). "Ensembles of incremental learners to detect anomalies in ad hoc sensor networks". ad hoc networks, $35,14-36$.

Capozzoli, A., Lauro, F. and Khan, I. (2015). "Fault detection analysis using data mining techniques for a cluster of smart office buildings." Expert Systems with Applications, 42(9), 4324-4338.

de Angelis, M., Patelli, E., and Beer, M. (2016). "Forced Monte Carlo simulation strategy for the design of maintenance plans with multiple inspections." ASCE-ASME Journal of Risk and Uncertainty in Engineering Systems, Part A: Civil Engineering, 3(2), D4016001. 
De Oca, V.M., Jeske, D.R., Zhang, Q., Rendon, C. and Marvasti, M., 2010. A cusum changepoint detection algorithm for non-stationary sequences with application to data network surveillance. Journal of Systems and Software, 83(7), pp.1288-1297.

Ding, L. and Drogemuller, R. (2009). "Towards sustainable facilities management." Proceedings of Technology, Design and Process Innovation in the Built Environment, Spon Press, 399-418.

Eastman, C., Teicholz, P., Sacks, R. and Liston, K. (2011). BIM Handbook: a Guide to Building Information Modeling for Owners, Managers, Designers, Engineers and Contractors. Wiley, Hoboken, NJ, USA.

Fan, C., Xiao, F., Li, Z. and Wang, J. (2018). "Unsupervised data analytics in mining big building operational data for energy efficiency enhancement: A review." Energy and Buildings, 159, 296-308.

Ferraz, G. T., De Brito, J., De Freitas, V. P., and Silvestre, J. D. (2016). "State-of-the-art review of building inspection systems." Journal of performance of constructed facilities, 30(5), 04016018.

Fryzlewicz, P., (2014). "Wild binary segmentation for multiple change-point detection". The Annals of Statistics, 42(6), 2243-2281.

Gao, X., and Pishdad-Bozorgi, P. (2019). "BIM-enabled facilities operation and maintenance: A review". Advanced engineering informatics, 39, 227-247.

Guo, L., and Wang, Y. H. (2013). "The Preliminary Study for the Fault Diagnosis of EMU Based on Expert System.” Applied Mechanics and Materials, Trans Tech Publications, 409, 1453-1460.

Hamledari, H., Rezazadeh Azar, E., and McCabe, B. (2017). "IFC-based development of asbuilt and as-is BIMs using construction and facility inspection data: Site-to-BIM data transfer automation." Journal of Computing in Civil Engineering, 32(2), 04017075.

Hautefeuille, M., O’Mahony, C., O'Flynn, B., Khalfi, K. and Peters, F. (2008). “A MEMSbased wireless multisensor module for environmental monitoring." Microelectronics Reliability, 48(6), 906-910.

Horrigan, M., Turner, W.J. and O’Donnell, J., (2018). “A statistically-based fault detection approach for environmental and energy management in buildings". Energy and Buildings, 158, 1499-1509.

Hou, L., Wang, Y., Wang, X., Maynard, N., Cameron, I. T., Zhang, S., and Jiao, Y. (2014). "Combining photogrammetry and augmented reality towards an integrated facility management system for the oil industry." Proceedings of the IEEE, 102(2), 204-220.

Jalori, S. and Reddy, T.A. (2015). "A New Clustering Method to Identify Outliers and Diurnal Schedules from Building Energy Interval Data.” ASHRAE Transactions, 121(2).

Korolija, I., Zhang, Y., Marjanovic-Halburd, L. and Hanby, V.I., (2013). "Regression models for predicting UK office building energy consumption from heating and cooling demands." Energy and Buildings, 59, 214-227.

Li, X., Wu, P., Shen, G. Q., Wang, X., and Teng, Y. (2017). Mapping the knowledge domains of Building Information Modeling (BIM): A bibliometric approach. Automation in Construction, 84, 195-206.

Li, J., Panchabikesan, K., Yu, Z., Haghighat, F., El Mankibi, M. and Corgier, D., (2019). "Systematic data mining-based framework to discover potential energy waste patterns in residential buildings." Energy and Buildings, 199, 562-578.

Li, Q., Meng, Q., Cai, J., Yoshino, H. and Mochida, A., (2009). "Applying support vector machine to predict hourly cooling load in the building." Applied Energy, 86(10), 22492256. 
Lu, Q., Chen, L., Lee, S. and Zhao, X. (2018). "Activity theory-based analysis of BIM implementation in building O\&M and first response." Automation in Construction, 85, 317-332, https://doi.org/10.1016/j.autcon.2017.10.017.

Lu, Q., Parlikad, A., Woodall, P., Don Ranasinghe, G., and Heaton, J. (2019). "Developing a dynamic digital twin at a building level using Cambridge campus as a case study." International Conference on Smart Infrastructure and Construction, Cambridge, UK. https://doi.org/10.17863/CAM.38523

Lu Q., Xie X., Heaton J., Parlikad A.K., and Schooling J. (2020a). "From BIM Towards Digital Twin: Strategy and Future Development for Smart Asset Management." Studies in Computational Intelligence, vol 853. Springer, Cham.

Lu, Q., Parlikad, A.K., Woodall, P., Ranasinghe, G.D., Xie, X., Liang, Z., Konstantinou, E., Heaton, J. and Schooling, J. (2020b). "Developing a Digital Twin at Building and City Levels: Case Study of West Cambridge Campus." Journal of Management in Engineering, 36(3), 05020004.

Memarzadeh, M., and Pozzi, M. (2016). "Integrated inspection scheduling and maintenance planning for infrastructure systems." Computer-Aided Civil and Infrastructure Engineering, 31(6), 403-415.

Memoori. (2014). "Big Data for Smart Buildings 2015 to 2020 Market Prospects." working paper, Meemoori Research AB, Stockholm Sweden.

Motamedi, A., Hammad, A. and Asen, Y., 2014. Knowledge-assisted BIM-based visual analytics for failure root cause detection in facilities management. Automation in construction, 43, 73-83.

Mustafaraj, G., Lowry, G. and Chen, J., (2011). "Prediction of room temperature and relative humidity by autoregressive linear and nonlinear neural network models for an open office." Energy and Buildings, 43(6), 1452-1460.

National Infrastructure Commission (NIC) (2017). "Data for the Public Good." $<$ https://www.nic.org.uk/wp-content/uploads/Data-for-the-Public-Good-NICReport.pdf> (Dec. 30, 2018).

Pena, M., Biscarri, F., Guerrero, J.I., Monedero, I. and León, C. (2016). "Rule-based system to detect energy efficiency anomalies in smart buildings, a data mining approach." Expert Systems with Applications, 56, 242-255.

Pereira, P.F. and Ramos, N.M., (2018.) "Detection of occupant actions in buildings through change point analysis of in-situ measurements." Energy and Buildings, 173, 365-377.

Pishdad-Bozorgi, P., Gao, X., Eastman, C. and Self, A.P. (2018). "Planning and developing facility management-enabled building information model (FM-enabled BIM)." Automation in Construction, 87, 22-38.

Schneider, R., Rogge, A., Thöns, S., Bismut, E., and Straub, D. (2018). “A sampling-based approach to identifying optimal inspection and repair strategies for offshore jacket structures." Proc. 6th International Symposium on Life-Cycle Civil Engineering (IALCCE), Ghent.

Shou, W., Wang, J., Wu, P., and Wang, X. (2020). "Value adding and non-value adding activities in turnaround maintenance process: classification, validation, and benefits." Production Planning \& Control, 31(1), 60-77.

Sun, B., Luh, P.B., Jia, Q.S., O'Neill, Z. and Song, F., (2013). "Building energy doctors: An SPC and Kalman filter-based method for system-level fault detection in HVAC systems." IEEE Transactions on Automation Science and Engineering, 11(1), 215-229.

Vellei, M., Herrera, M., Fosas, D. and Natarajan, S. (2017). "The influence of relative humidity on adaptive thermal comfort." Building and Environment, 124, 171-185.

Wang, H. and Chen, Y., (2016). "A robust fault detection and diagnosis strategy for multiple faults of VAV air handling units". Energy and Buildings, 127, 442-451. 
Wang, P., Wu, P., Wang, X., Chen, X., and Zhou, T. (2020). "Developing optimal scaffolding erection through the integration of lean and work posture analysis." Engineering, Construction and Architectural Management, https://doi.org/10.1108/ECAM-04-20190193.

Wijayasekara, D., Linda, O., Manic, M. and Rieger, C. (2014). "Mining building energy management system data using fuzzy anomaly detection and linguistic descriptions." IEEE Transactions on Industrial Informatics, 10(3), 1829-1840.

$\mathrm{Wu}$, S. and Clements-Croome, D. (2007). "Understanding the indoor environment through mining sensory data-A case study." Energy and Buildings, 39(11), 1183-1191.

Yu, Y., Woradechjumroen, D., and Yu, D. (2014). "A review of fault detection and diagnosis methodologies on air-handling units." Energy and Buildings, 82, 550-562.

Zhan, J., Ge, X. J., Huang, S., Zhao, L., Wong, J. K. W., and He, S. X. (2019). "Improvement of the inspection-repair process with building information modelling and image classification." Facilities, 37(7/8), 395-414. 